Perception of Object Persistence: The Origins of Object Permanence in Infancy

\author{
Gavin Bremner \\ Lancaster University \\ Alan Slater \\ Exeter University \\ Scott Johnson \\ University of California, Los Angeles
}

Key words: [AU: Please add key words here] 


\begin{abstract}
A dominant account of object knowledge in infancy is based on the assumption that infants possess innate core knowledge of objects through which they reason about events and look longer at those that violate their expectations on the basis of this knowledge. In this article, we propose a perceptual model in which younger infants' perception of object persistence is subject to greater perceptual constraints compared with infants a few months older, and in which young infants require many cues to perceive object persistence across occlusion. Young infants perceive object persistence under limited conditions and over the early months, perception of persistence becomes more robust. The same analysis may be applied to cases in which stationary objects are occluded, including tasks assessing infants' numerical competence. We argue that these perceptual developments within the first 6 months likely underpin the later development of cognitive principles, including object permanence.
\end{abstract}


Investigation of the development of object permanence in infancy started with Piaget's constructionist account (1), according to which infants progressively construct an objective knowledge of the world, with permanence beginning to emerge around 8 to 9 months, when infants begin to search for hidden objects. More recently, investigators developed ways to test young infants' responses to hidden objects that did not rely on searching for objects, and these experiments led to the view that knowledge of the physical world is innate. Specifically, infants possess innate core knowledge and reason about the events that they encounter (2-4). A key aspect of innate knowledge is the principle of object persistence (5).

This account is based largely on experiments that measure the time infants look at objects, with longer looks at events that break physical laws interpreted as evidence that infants' expectations have been violated (the Violation of Expectancy Technique: VoE). These trials generally follow familiarization to a lawful event, and in this sense, the VoE technique is similar structurally to Habituation-Recovery and Habituation-Novelty techniques that investigate perceptual abilities in infants starting at birth (6). However, the similarity of the measures leads to interpretative problems for the $\mathrm{VoE}$ technique, because experiments must rule out lower-level perceptual bases for longer looking at the violation test trial (e.g. 7). Investigators have been ingenious in this respect. Nevertheless, we argue that it is often difficult to identify the lower-level principles that may drive infants' looking. Additionally, we contend that relatively low-level perceptual principles form the developmental origins of later knowledge, and longer looking in VoE tasks reflects detection of a perceptual discrepancy rather than reasoning about the event.

Much of the evidence on which claims about innate knowledge are based is from experiments with infants starting at 3 or 4 months. Stronger evidence for innateness would involve measuring newborn ability, but this kind of work is rare. This leaves a gap of several months during which infants could develop awareness of the world. In this review, we 
demonstrate that important development occurs during the first 6 months that likely forms a perceptual basis for the knowledge that nativists identify as innate. We are not denying the presence of innate ability, but rather believe that a primarily perceptual ability provides a platform for conceptual knowledge emerging later in infancy.

Our account stems from work by Gibson (8) and Michotte (9). When an object disappears behind an occluder, several perceptual cues specify its continuity. Young infants typically do not interpret these cues efficiently so they need a combination of cues to perceive object persistence. Additionally, perception of persistence is subject to spatio-temporal constraints, only appearing across relatively small gaps in perception. However, developments occur during the first 6 months after birth, resulting in robust perception of object persistence. Therefore, rather than assuming an innate principle of object persistence, we argue for an approach that emphasizes the conditions under which infants detect persistence in the events they perceive, and considers how these conditions change with age. Additionally, data gathered from VoE experiments can be interpreted as increased attention to an event in which infants perceive persistence, which is later violated. Thus, we argue that interpretations that invoke reasoning on the part of the infant are unnecessary. Although nativist accounts might explain our data in terms of perceptual constraints on the circumstances under which infants display innate knowledge, our account is parsimonious in explaining development of object persistence in terms of perceptual abilities, without invoking knowledge states and reasoning.

\section{Object Unity}

In one study (10), 4-month-olds perceived a rod as complete even though its central section was occluded (Figure 1). Infants were habituated to a rod moving laterally behind an occluder, then saw two test displays with the occluder missing: either the previously visible 
rod parts or a complete rod. If infants had perceived a complete rod during habituation, they should prefer the novel disconnected rod parts. Infants showed this preference, whereas a control group habituated to a display in which only the top rod part moved showed no preference. [AU: It seems the verb in the previous sentence should be either "moved" OR "showed no preference" (not both). Please clarify.]

Although this finding might be interpreted as evidence for object permanence, and indeed the researchers suggested that it is based on an innate notion that objects are coherent and persistent, it can also be interpreted in terms of perceptual completion. A perceptual account seems appropriate because further work indicated various perceptual factors that determine detection of unity. Object unity is only perceived when the rod is in motion (10, Experiment 2), apparently because motion provides a depth cue by producing deletion and accretion of background $(11,12)$. Additionally, unity is only perceived when rod parts are perceptually relatable, or aligned (11), and is influenced by the Gestalt principle of figural goodness (13). Finally, object unity is hard to elicit at birth (14), appearing only under simplified stroboscopic presentation conditions $(15,16)$, and beginning to appear in the task at around 2 months when the occluder is narrow or contains gaps (17). Thus, robustness of object unity increases over the early months and several perceptual variables influence its perception.

\section{Trajectory Continuity}

\section{A Habituation Novelty Approach}

Trajectory continuity could involve core knowledge of permanence applied to moving objects, but a perceptual account appears appropriate. In one study (18), 4-month-olds were habituated to a ball that cycled back and forth, then disappeared behind a rectangular occluder in the central section of its trajectory. On test trials, the occluder was removed and 
the ball either moved discontinuously, disappearing and reappearing where the edges of the occluder had been during habituation, or moved continuously (Figure 2). If infants perceived the ball's trajectory as continuous during habituation, they should look more [AU: longer?] at the discontinuous display as a novel event, whereas if they perceived discontinuity, they should look more [AU: longer?] at the continuous display. Four-month-olds preferred the continuous display, indicating that they had perceived the habituation trajectory as discontinuous. In contrast, 6-month-olds preferred the opposite, perceiving continuity in the habituation trajectory.

In a second experiment, 2- and 4-month-olds saw an event in which the occluder was slightly wider than the ball (Figure 3a). Under these conditions, 4-month-olds perceived continuity, but 2-month-olds showed no preference between test displays. Thus, when the perceptual load associated with filling in the gap in perception is minimal, 4-month-olds perceived a continuous trajectory, whereas 2-month-olds did not. For 4-month-olds at any rate, trajectory perception depends on the width of the occluder. Another experiment revealed that perception of trajectory continuity increased linearly as a function of the width of the occluder, rather than shifting from perceiving discontinuity to perceiving continuity abruptly at some key occluder width. The strength of the trajectory percept ranges continuously from perception of discontinuity, to perception of continuity, though an intermediate state of no percept either way, suggesting a perceptual basis for performance. Perhaps core knowledge is only strong enough to emerge when perceptual load is low, but parsimony favors a purely perceptual account in which young infants' ability to perceive continuity is constrained by spatio-temporal factors.

\section{Predictive Tracking and Trajectory Continuity}


Predictive tracking is also used to measure perception of object continuity. Infants are presented with an occlusion event. Their tracking of the object is measured by an eyetracker, with anticipatory tracking of its re-emergence from behind the occluder evidence that they perceive object continuity. Convergent evidence arises from this sort of work, including growing anticipatory tracking with age $(19,20)$. In a wide occluder-tracking task $(21), 4$ month-olds did not predictively track consistently, whereas 6-month-olds predicted at a higher level, in keeping with the first experiment (18). Additionally, 4-month-olds benefited from prior exposure to a continuous trajectory with no occluder $(21,22)$, a finding that strengthens an experience-based perceptual development account of the emergence of object continuity. The agreement between measures increases confidence that the age-related improvement in anticipatory tracking is not simply due to improved oculomotor control. And although, like the habituation-novelty work, the work in Johnson's lab used 2D animations, similar anticipatory tracking data emerge when a 3D display is used (23). Thus, the anticipatory tracking data appear robust and the convergence of results with the habituationnovelty work backs a perceptual account.

\section{Spatiotemporal Constraints on Perception of Continuity}

Four-month-olds could perceive trajectory continuity when the occluder is narrow because the object is out of sight for a short time or because it is out of sight across a short distance. To tease apart these factors, in one study (24), researchers first increased the ball diameter until it was almost as wide as the wide occluder in a prior study (18; Figure 3b). This resulted in a short temporal gap but a large spatial gap in the trajectory. Under these circumstances, 4-month-olds perceived trajectory continuity, suggesting that time out of sight was key. However, in another experiment, the speed of a small ball was increased while it was behind the wide occluder, or decreased while it was behind a narrow occluder. Both 
manipulations resulted in perception of continuity, so we conclude that 4-month-olds perceive trajectory continuity when the perceptual load is reduced by limiting either the temporal or the spatial gap over which the trajectory must be interpolated.

Further investigation indicated that 4-month-olds no longer perceived continuity following a change in the object's trajectory, from a high to a low horizontal trajectory (Figure 3c) or from a falling to a rising diagonal trajectory (Figure 3d;25). And in the case of the falling-rising trajectory, this occurred even if a surface was included in the scene to explain change from falling to rising trajectory through a hidden collision (Figure 3e). Another constraint applies to perception of linear trajectories: Although 4-month-olds perceive trajectory continuity for horizontal and vertical trajectories (26), their ability to perceive continuity of diagonal trajectories is limited (Figure 3f; 25, 26). In contrast, 6month-olds perceive continuity for all trajectory orientations, and it is likely that the limit seen at 4 months is due to difficulty tracking oblique movements (and hence in perceiving their continuity) because of the need to coordinate input to extra-ocular muscles controlling vertical and horizontal components of movement (27). In an eye-tracking experiment, (28) researchers detected errors in infants' circular tracking; similar errors could affect oblique tracking because it requires similar coordination of extra-ocular muscles.

\section{Multisensory Information Supports Perception of Continuity}

Supplementing information about object movement in habituation trials with congruent auditory information supported perception of trajectory continuity across occluder widths that yielded negative or null results in unisensory presentation (29)[AU: Please revise/simplify the previous sentence so that its meaning is clearer.] Even providing a static continuous sound provided some information for continuity, likely because the sound was continuous across the gap in visual perception. However, only adding dynamic congruent 
sound apparently enhances 4-month-olds' anticipatory tracking (30), so the effect of multisensory information on tracking and perception of continuity needs further investigation.

\section{Visual Cues to Occlusion and Trajectory Continuity}

For adults, deletion and accretion at virtual edges with no visible occluder is sufficient to create the tunnel effect (31): the percept of a moving object disappearing and reappearing as if passing through an invisible slit in a surface $(9,32)$. Deletion and accretion seem to be important cues to object persistence for infants, because 5- to 9-month-olds' predictive tracking was reduced if, instead of undergoing deletion and accretion, the object underwent instantaneous disappearance/reappearance or implosion/explosion at the occluder boundaries (33). However, although deletion and accretion may be necessary cues for perceiving object persistence, successful predictive tracking when these cues exist on each side of an occluder improves with age (19). Also, these cues do not appear to be sufficient to specify occlusion for young infants, or our discontinuous test display would appear similar to the habituation display and would attract low levels of looking. In other words, if 4-month-olds perceived our discontinuous test display as an occlusion event, we would not have obtained strong preferences for the discontinuous event.

We investigated which additional cues were required to specify an occlusion event to 4-month-olds (34). First, we investigated infants' perception of the Kanizsa figure (35) as an occluding surface. This figure creates the percept of a rectangular surface because the inducing elements are perceived as circles that are partly occluded by the surface. Thus, it embodies two cues: occluding edges and occlusion of background. Eight-month-olds, but apparently not 5-month-olds, perceived the illusory Kanizsa rectangle as an occluding surface (36). However, newborns perceive illusory surfaces (37), and the illusory surface in this study was quite wide, leading to a relatively long occlusion time. So we manipulated 
occluder width in the case of the Kanizsa illusory surface (Figure 4a and $4 \mathrm{~b}$ ). When the Kanizsa figure was narrow, 4-month-olds treated it as an occluding surface and perceived trajectory continuity. Next, we tested the separate effectiveness of two cues that exist in the case of a visible occluder and the Kanizsa figure: a visible edge (Figure 4c) and background occlusion (Figure 4d). Besides accretion and deletion, neither of those cues was sufficient when presented on its own, but presentation of both (still with no visible, luminance-defined surface) was sufficient to support perception of trajectory continuity.

The Kanizsa figure effect indicates that occlusion cues need not be spatially contiguous with the path of object motion, and this raises the question of whether accretiondeletion cues and occluding surface cues need to be congruent. Apparently they do, because infants did not perceive trajectory continuity in events in which accretion deletion occurred at a point earlier or later in the trajectory than it should (Figure 5a and 5b), or as if at an oblique occluding edge when the real occluding edges were vertical (Figure 5c; 38).

\section{Summary: Trajectory Continuity}

Besides pointing to young infants' need for many cues to occlusion, our work indicates perceptual constraints (such as limited time or distance out of sight, difficulty with oblique trajectories) on 4-month-olds' detection of trajectory continuity that speaks for a perceptual account of object persistence. Evidence suggests gradual development in which infants progressively perceive object persistence across larger spatial and temporal gaps and across an increasing range of trajectories. Studies using anticipatory tracking as a measure of object persistence suggest similar perceptual constraints and gradual development[AU: Please confirm that my edits haven't changed the meaning of the previous sentence.] (for a review, see 39). Such findings do not mesh with accounts that propose innate knowledge of permanence. Additionally, we see no need to assume that infants reason about events. 
Instead, longer looking may simply indicate detection of a discontinuity or perturbation in the perceptual flow of events with which infants have become familiar.

\section{Extending the Analysis to Occlusion of Stationary Objects}

This analysis should also be possible in the case of stationary object occlusion. Researchers should investigate the conditions for perception of persistence of a stationary object occluded and revealed by an occluder moving laterally across the object's location. Perception of persistence may be stronger in the case of stationary objects occluded than objects moved behind an occluder. For instance, in one study, representations created from direct perception of the object were more robust than those created from inferring the location of an object placed behind a screen (40). In another study, with older infants, infants of 10 months and older were more likely to search for an object that was placed before hiding than for one that moved behind an occluder (41). An advantage in perceiving persistence of a stationary object seems likely because the final resting place of a moving object that disappears behind an occluder is specified less precisely than the position of one seen resting in a given place before occlusion.

Such work may provide an alternative interpretation of classic nativist investigations such as the drawbridge study $(42,43)$, in which infants reason that a rising drawbridge cannot rotate through a stationary solid obstruction. Rather than claiming that infants represent the existence of an obstructing object, we may be able to demonstrate that infants perceive the persistence of this stationary object through the perceptual cues specifying its progressive occlusion by the drawbridge. Thus, although longer looking at the impossible event indicates detection of a violation, this is detection of a perceptual anomaly that would occur if two fully visible objects moved through each other. 
This type of analysis may be extended to studies on addition and subtraction $(44,45)$ in which, when an object is added or removed from behind an occluder, infants looked longer at the incorrect numerical outcome when the occluder was removed. Our account is in keeping with alternative explanations of infants' responses to numerical violations based on object tracking and object files (40, 46-48). Infants attach attentional pointers (files) to objects as they are introduced, and track them as they are occluded, added, or removed from behind the occluder. Longer looking at incorrect numerical outcomes, rather than reflecting numerical knowledge, reflects detection of a perceptual violation of objecthood, specifically detection that an object seen removed from behind the occluder is still there, or an object seen introduced behind the occluder is not there. In our analysis, the infant's response does not demand knowledge of number or the ability to reason. Supporting this idea, in the subtraction condition (49), eye-tracker evidence indicated that infants looked longer specifically at the object that was seen removed but was still present (49).

\section{Looking Ahead}

Research reported here supports a perceptual account of object persistence. Rather than advance object persistence as an innate principle though which events are interpreted, we propose that persistence is specified by perceptual events such as deletion and accretion, and the developmental question is about infants' changing ability to perceive object persistence on the basis of these cues. Four-month-olds perceive persistence across shorter spatial and temporal gaps than 6-month-olds, and they require more cues to specify occlusion (and hence persistence) than adults. Researchers should test our prediction that as infants develop, they need progressively fewer cues to detect object persistence. Also, we need to establish the cues infants use to perceive persistence of stationary objects hidden by occluders. The principles of physical reality exist in information in the world, and development in early 
infancy is primarily about increasing the efficiency of detection of this information. The question of when and how infants develop a mental concept of object permanence remains, but such knowledge likely is rooted in perception of object persistence. 


\section{Authors' Note}

[AU: Please add contact information here, as well as any acknowledgments you wish to include.] 


\section{References}

1. Piaget, J. (1954). The construction of reality in the child. (trans M. Cook) New York, NY: Basic Books (originally published in French, 1936).

2. Baillargeon, R. (1992). The object concept revisited: new directions in the investigations of infants' physical knowledge. In C.E. Granrud (Ed.), Visual perception in infancy. Carnegie-Mellon Symposia on Cognition, Vol. 23, Hillsdale, NJ: Erlbaum. [AU: Please provide page numbers for the chapter.]

3. Spelke, E.R., Breinlinger, K., Macomber, J., \& Jacobson, K. (1992). Origins of knowledge. Psychological Review, 99, 605-632.

4. Spelke, E.S., \& Kinzler, K.D. (2007). Core knowledge. Developmental Science, 10, 89-96.

5. Baillargeon, R. (2008). Innate ideas revisited: For a principle of persistence in infants' physical reasoning. Perspectives on Psychological Science, 3, 2-13.

6. Bornstein, M. H. (1985). Habituation of attention as a measure of visual information processing in human infants: Summary, systematization, and synthesis. In G. Gottlieb \& N. A. Krasnegor (Eds.), Measurement of audition and vision in the first year of postnatal life: A methodological overview (pp. 253-300). Norwood, NJ: Ablex.

7. Cohen, L. B. (2004). Uses and misuses of habituation and related preference paradigms. Infant and Child Development, 13, 349-352.

8. Gibson, J.J. (1979). The ecological approach to visual perception. Boston, MA: Houghton Mifflin.

9. Michotte A., Thines, G., \& Crabbe, G. (1964/1991). Les complements amodaux des structures perceptives [AU: Please translate French into English]. Louvain, Belgium: Publications Universitaires. Excerpted in G. Thines, A. Costall, \& G. Butterworth (Eds.), Michotte's experimental phenomenology of perception (pp. 140-167). Hillsdale, NJ: Erlbaum, 1991. 
10. Kellman, P.J., \& Spelke, E.R. (1983). Perception of partly occluded objects in infancy. Cognitive Psychology, 15, 483-524

11. Johnson, S. P., \& Aslin, R. N. (1996). Perception of object unity in young infants: The roles of motion, depth, and orientation. Cognitive Development, 11, 161-180.

12. Johnson, S. P., \& Náñez, J. E. (1995). Young infants' perception of object unity in twodimensional displays. Infant Behavior and Development, 18, 133-143.

13. Johnson, S.P., Bremner, J.G., Slater, A.M., \& Mason, U.C. (2000) The role of good form in infants' perception of partly occluded objects, Journal of Experimental Child Psychology, 76, 1-25.

14. Slater, A., Johnson, S. P., Brown, E., \& Badenoch, M. (1996). Newborn infants' perception of partly occluded objects. Infant Behavior and Development, 19, 145148

15. Valenza, E., \& Bulf, H. (2011). Early development of object unity: Evidence for perceptual completion in newborns. Developmental Science, 14, 799-808.

16. Valenza, E., Leo, I., Gava, L., \& Simion, F. (2006). Perceptual completion in newborn human infants. Child Development, 77, 1810-1821.

17. Johnson, S. P., \& Aslin, R. N. (1995). Perception of object unity in 2-month-old infants. Developmental Psychology, 31, 739-745

18. Johnson, S.P., Bremner, J.G., Slater, A.M., Mason, U.C., Foster, K., \& Cheshire, A. (2003). Infants' perception of object trajectories, Child Development, 74, 94-108.

19. Bertenthal, B.I., Gredebäck, G., \& Boyer, T.W. (2013). Differential contributions of development and learning to infants' knowledge of object continuity and discontinuity. Child Development, 84, 413-421.

20. Gredebäck, G., \& von Hofsten, C. (2004). Infants' evolving representations of object motion during occlusion: A longitudinal study of 6- to 12-month-old infants. Infancy, 
6, 165-184.

21. Johnson, S. P., Amso, D., \& Slemmer, J. A. (2003). Development of object concepts in infancy: Evidence for early learning in an eye-tracking paradigm. Proceedings of the National Academy of Sciences (USA), 100, 10568-10573.

22, Johnson, S. P., \& Shuwairi, S. M. (2009). Learning and memory facilitate predictive tracking in 4-month-olds. Journal of Experimental Child Psychology, 102, 122-130.

23. Johnson, S.P., Bremner, J.G., Slater, A.M., Shuwairi, S.M., Mason, U., Spring, J., \& Usherwood, B. (2012). Young infants' perception of the trajectories of two- and three-dimensional objects. Journal of Experimental Child Psychology, 113, 177-185.

24. Bremner, J.G., Johnson, S.P., Slater, A.M., Mason, U., Foster, K., Cheshire, A., \& Spring, J. (2005). Conditions for young infants' perception of object trajectories. Child Development, 76, 1029-1043.

25. Bremner, J.G., Johnson, S.P., Slater, A.M., Mason, U., Foster, K., Cheshire, A., \& Spring, J. (2007). Conditions for young infants' failure to perceive trajectory continuity. Developmental Science, 10, 613-624.

26. Bremner, J.G., Slater, A.M., Mason, U., Spring, J., \& Johnson, S.P. Four-month-old infants fail to perceive continuity of oblique trajectories. Unpublished manuscript under revision for Developmental Psychology. [AU: Has this paper been accepted for publication? If not, we will revise the cite to "unpublished manuscript."]

27. Schiller, P. H. (1998). The neural control of visually guided eye movements. In J. E. Richards (Ed.), Cognitive neuroscience of attention. A developmental perspective (pp. 3-50). Mahwah, NJ: Erlbaum.

28. Grönqvist, H., Gredebäck, G., \& von Hofsten, C. (2006). Developmental asymmetries between horizontal and vertical tracking. Vision Research, 46, 1754-1761. 
29. Bremner, J.G., Johnson, S.P., Slater, A.M., Mason, U., \& Spring, J. (2012). The effects of auditory information on 4-month-old infants' perception of trajectory continuity. Child Development, 83, 954-964.

30. Kirkham, N. Z., Wagner, J., Swan, K., \& Johnson, S. P. (2012). Sound support: Intermodal information facilitates infants' perception of an occluded trajectory. Infant Behavior \& Development, 35, 174-178.

31. Burke, L. (1952). On the tunnel effect. Quarterly Journal of Experimental Psychology, 4, 121-138.

32. Kahneman, D., Triesman, A., \& Gibbs, B.J. (1992). The reviewing of object files: Object-specific integration of information. Cognitive Psychology, 24, 174-219.

33. Bertenthal, B.J., Longo, M.R., \& Kenny, S. (2007). Phenomenal permanence and the development of predictive tracking in infancy. Child Development, 78, 350-363.

34. Bremner, J.G., Slater, A.M., Johnson, S.P., Mason, U. \& Spring, J. (2012). Illusory contour figures are perceived as occluding surfaces by four-month-old infants. Developmental Psychology, 48, 398-405.

35. Kanizsa, G. (1979). Organization in vision. New York, NY: Praeger.

36. Csibra, G. (2001). Illusory contour figures are perceived as occluding surfaces by 8month-old infants. Developmental Science, 4, F7-F11.

37. Valenza, E., \& Bulf, H. (2007). The role of kinetic information in newborns' perception of illusory contours. Developmental Science, 10, 492-501.

38. Bremner, J.G., Slater, A.M., Mason, U., Spring, J., \& Johnson, S.P. Perception of occlusion by young infants: Must the location and orientation of the occlusion event be congruent with the occluder? Unpublished manuscript under revision for Infancy.[AU: Has this paper been accepted for publication?]

39. Gredebäck, G., von Hofsten. C., \& Boudreau, J. P. (2002). Infants' visual tracking of 
continuous circular motion under conditions of occlusion and non-occlusion. Infant Behavior and Development, 25, 161-182.

40. Uller, C., Carey, S., Huntley-Fenner, G., \& Klatt, L. (1999). What representations might underlie infants' numerical knowledge? Cognitive Development, 14, 1-36.

41. Moore, M.K., \& Meltzoff, A.N. (1999). New findings on object permanence: A developmental difference between two types of occlusion. British Journal of Developmental Psychology, 17, 563-584.

42. Baillargeon, R., Spelke, E.S. \& Wasserman, S. (1985). Object permanence in fivemonth-old infants, Cognition, 20, 191-208.

43. Baillargeon, R. (1987a) Object permanence in 3.5- and 4.5-month-old infants, Developmental Psychology, 23, 655-664.

44. Wynn, K. (1992) Addition and subtraction by human infants. Nature, 358, 749-750.

45. Wynn, K. (1995) Origins of numerical knowledge. Mathematical Cognition, 1, 35-60.

46. Cheries, E.W., Wynn, K., \& Scholl, B.J. (2006). Interrupting infants' persisting object representations: An object-based limit? Developmental Science, 9, F50-F58.

47. Simon, T.J. (1997). Reconceptualizing the origins of number knowledge: A 'nonnumerical' account. Cognitive Development, 12, 349-372.

48. Simon, T.J. (1998). Computational evidence for the foundations of numerical competence. Developmental Science, 1, 71-78.

49. Bremner, J.G., Slater, A.M., Hayes, R.A., Mason, U., Murphy, C., Spring, J., \& Johnson, S.P. (2014). Eye-tracking infants in addition and subtraction tasks. Unpublished manuscript under revision for Journal of Experimental Child Psychology. [AU: Has this paper been accepted for publication?] 


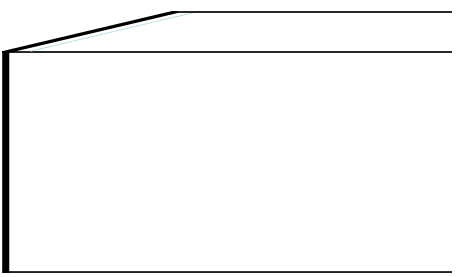

Figure 1. Displays presented by Kellman and Spelke (10). A. Habituation rod and box display B. Incomplete test display (visible rod parts) C. Complete test display 
Figure 2. Events presented by Johnson, Bremner et al. (18). A. The habituation display B. The discontinuous test display $\mathrm{C}$. The continuous test display 

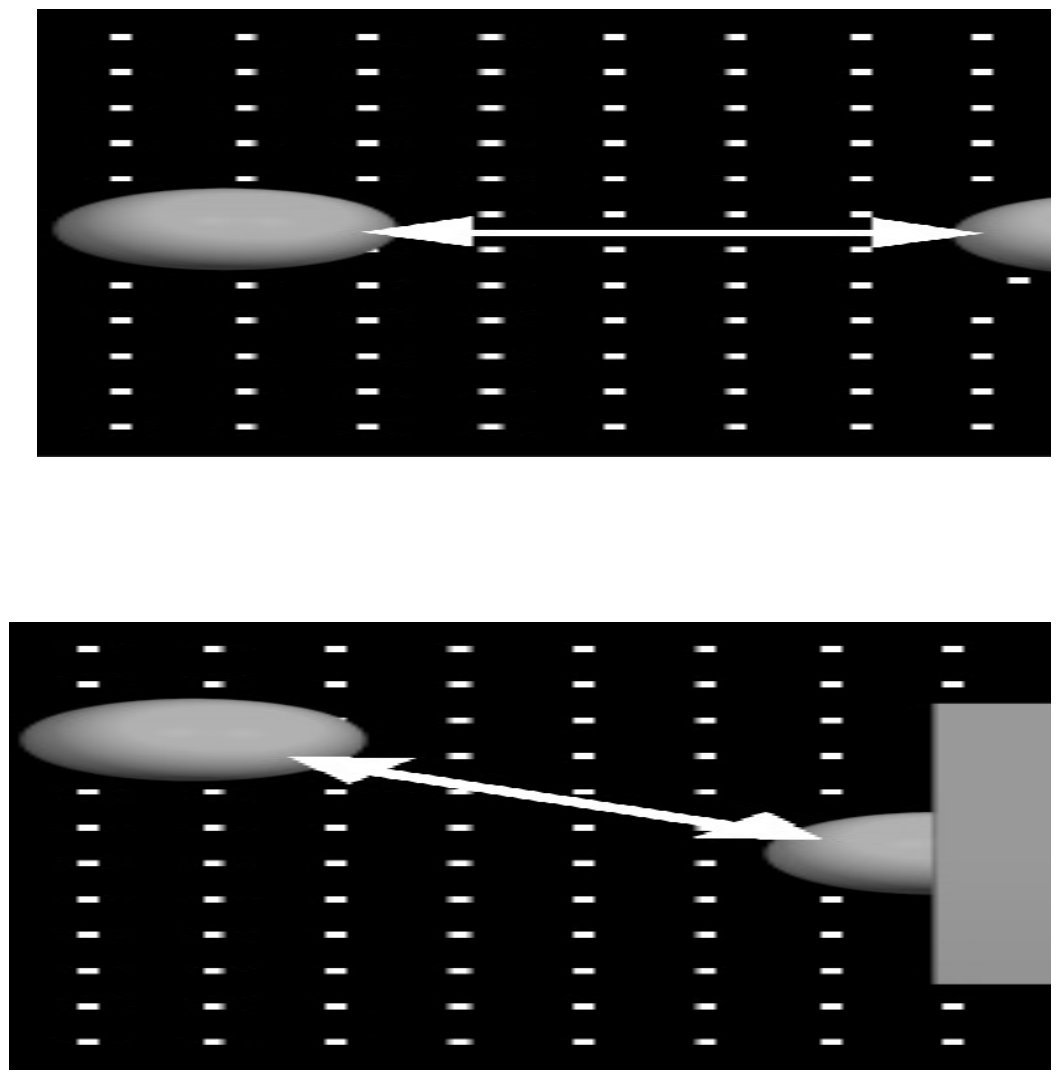

Figure 3. Habituation events. A. Narrow occluder event in Johnston, Bremner, et al. (18) Large ball (B) High-low trajectory (C), falling rising trajectory (D), falling-rising trajectory with surface (E), and linear oblique trajectory (F) events in Bremner et al. (2005) 
Figure 4. Habituation events in Bremner et al. (29). A. Wide Kanizsa B. Narrow Kanizsa C. Visible occluding edge but no background occlusion D. Background occlusion but no visible occluding edge 
Figure 5. Habituation events in Bremner et al. (26). A. Early occlusion B. Late occlusion C. Oblique edge occlusion 